\title{
Secure Hotspot with Multi-Factor Authentication through NFC on Smartphones
}

\author{
Wiphop Pomak ${ }^{1}$, Yachai Limpiyakorn ${ }^{1+}$ \\ ${ }^{1}$ Department of Computer Engineering, Chulalongkorn University, Bangkok 10330, Thailand
}

\begin{abstract}
Many today enterprises attempt to protect their wireless networks from security threats. Another layer of security is typically added to verify users when connecting to a wireless network. One of the wellknown enterprise authentication methods includes WPA2 Enterprise with 802.1X standard, which uses the EAP framework to enable user authentication. Deploying WPA2-Enterprise requires a RADIUS server for handling the task of authenticating access. However, credentials management for all users is a hassle for administrators. Strong authentication processes also require more and more certain actions from users. To enhance security for both users and wireless networks, this research thus presents an approach of Wi-Fi hotspot authentication by adopting MFA technique in addition to NFC utilization for credential information exchange. The implementation is developed on NFC-enabled Android smartphones through network model based on WPA2-802.1X authentication with EAP-TLS. The benefits of the proposed method would be the ease of Wi-Fi configuration and simplicity of internet access as reported in the experimental result.
\end{abstract}

Keywords: near field communication, multi-factor authentication, wireless network security.

\section{Introduction}

With the advent of smartphones and tablet devices, many people conduct their online activities through wireless access including cellular network or Wi-Fi. Securing mobile has become more concern as mobile phone traffic is more handled by Wi-Fi hotspots than by cellular networks [1]. This leads the phones to take more risks especially in the enterprises with a Bring Your Own Device (BYOD) policy [2]. Employees can bring their own portable devices to work and use them to access corporate resources such as email, file servers, databases, cloud, and software. Difficulties to manage the BYOD are monitoring and controlling access to corporate networks and private networks that may lead to data leakage [3]. Consequently, enterprises have considered internet access authentication as the first essential matter to protect their Wi-Fi networks from security threats. Today traditional authentication methods such as user/password, or even WiFi Protected Access (WPA) and WPA2 are still open to attack. The WPA/WPA2-Enterprise and RADIUS (802.1X) with Extensible Authentication Protocol (EAP) especially EAP Transport Layer Security (EAPTLS) method is introduced as the maximum-security level for corporate Wi-Fi networks today [4]. EAP-TLS uses a public key infrastructure (PKI) to authorize a client and server through certificates issued by a trusted certificate authority (CA) for mutual identification. Although the method is very secure, deployments are difficult because digital certificates must be installed for each wireless device. If these pre-provision steps, such as certificate setup, can be rapidly configured, it is then feasible to address a user's burden on a small device as well as to support network security policies.

This paper presents an approach of automated Wi-Fi hotspot authentication to enhance security and simplicity for both clients and networks. The method adopts Multi-Factor Authentication (MFA) technique and utilizes Near Field Communication (NFC) for exchanging credential information.

\section{Background}

\footnotetext{
+ Corresponding author. Tel.: +668 2218 6959; fax: +668 22186955 .
}

E-mail address: Yachai.L@chula.ac.th. 
Near Field Communication, or NFC is contactless communication technology that based on RFID standards in the high-frequency range. NFC operates at $13.56 \mathrm{MHz}$ on ISO/IEC 18000-3 air interfaces, with data transfer rates ranging from 106, 212 and $424 \mathrm{Kbits}$ per second [5]. It is designed to be a secure form of data exchange that can communicate in a few centimetres of another i.e. around $20 \mathrm{~cm}$ theoretically $(10 \mathrm{~cm}$ or less is practical), which can reduce the likelihood unwanted interception.

Multi-Factor Authentication, or MFA [6] is a security system that needs more than one method of authentication from independent categories of credentials to verify the user's identity for a login or other transactions. MFA combines two or more independent credentials, which is typically separated into 3 categories: 1) knowledge factor or what the user knows (password/identification); 2) possession factor or what the user has (security token); and 3) Inherence factor or what the user is (biometric verification).

IEEE 802.1X [7] is an IEEE standard framework for encrypting and authenticating a user who is trying to associate to a wired or wireless network. The framework consists of three parties: Supplicant is client devices, authenticator is ethernet switch or wireless access point, and authentication server is host running software supporting the RADIUS and EAP protocols e.g. TLS, TTLS, PEAP, MSCHAPV2, etc. IEEE 802.1X utilizes EAP to establish a secure tunnel between participants involved in an authentication exchange.

Public Key Infrastructure, or PKI [8] is a digital certificate providing mutual authentication between EAP client and the RADIUS server whether other party is indeed who it claims to be. PKI certificate creates by Certificate Authority (CA) or issuer. For Private Certificate Authority, it generally needs to install the 'Root Certificate' from provider's CA on each client before it will recognize private server certificate.

Managing confidential for authentication on smartphones to comply with higher level of security policies is challenging. Matos et al. [9] presented an approach of applying NFC to deal with Wi-Fi hotspot authentication by exchanging prior network configuration information based on a side-channel. However, as the obtained credentials data are static values, it is likely to be cracked by an offline brute force attack and hard to handle for many users. Sudar et al. [10] used Time-based One-Time Password (TOTP) to authenticate mobile Wi-Fi hotspot through two devices. One acts as an access point while the other device connects to the access point as client. The credentials data is dynamically generated and turn into Quick Response Code (QR code). Nevertheless, QR code is easily decoded. If there are enough samples, the algorithm of the password generator could be resolved, then.

\section{Implementation}

The implementation is developed on Android platform using a NFC-enabled smartphone (Motorola-G5 Plus) running Android 7.0 through Wi-Fi hotspot architecture based on WPA2/ 802.1x authentication with EAP-TLS method. The RADIUS authentication server is required for the design. The development consists of four main components as shown in Fig. 1, that is:

- Transceiver

A transceiver is an important module for data exchange between smartphones and NFC reader desktop. It requires an application running on both client and server-side. It relies on Host-based Card Emulation (HCE) mode and Application Data Protocol Unit (APDU) protocol to send commands and receive responses in hexadecimal sequences format as bidirected communication.

- Certificate manager

Certificate manager supports installing received certificates from the provider. It requires 2 certificates on the client device: user certificate and root certificate authority. This function is necessary for user privacy as setting up certificates without user's interaction could lead to serious consequent issues.

- Credential storage

Keystore system stores sensitive data such as certificates, identification, name, key, and other encrypted files. It mitigates the unauthorized use of key material. Asymmetric cryptography is used to encrypt data using public key before storing in the Keystore, while the private key is stored non-local on server-side. 


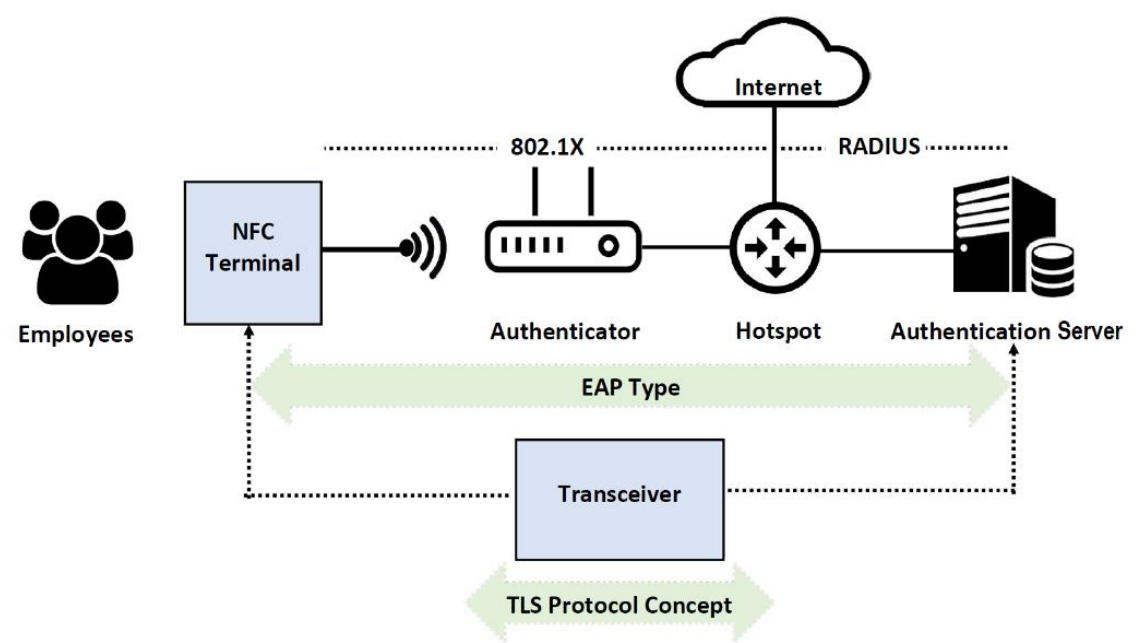

Fig. 1: Architectural design.

\section{- Network Connector}

This function facilitates secure connections to the target Access Point (AP) without further action required later for both of the certificates installed. Network configuration information will be provided by the authentication server including SSID, security types, EAP method, and certificates.

\section{Workflow}

In this work, NFC is utilized in exchange for network configuration information and MFA is applied to fortify security strength. The workflow of authentication process when users connect to the enterprise Wi-Fi hotspot is illustrated in Fig. 2. It is divided into four stages: 1) Unlock user's Keystore system, 2) Exchange credential data over NFC, 3) Set up certificates, and 4) Auto Wi-Fi connect.

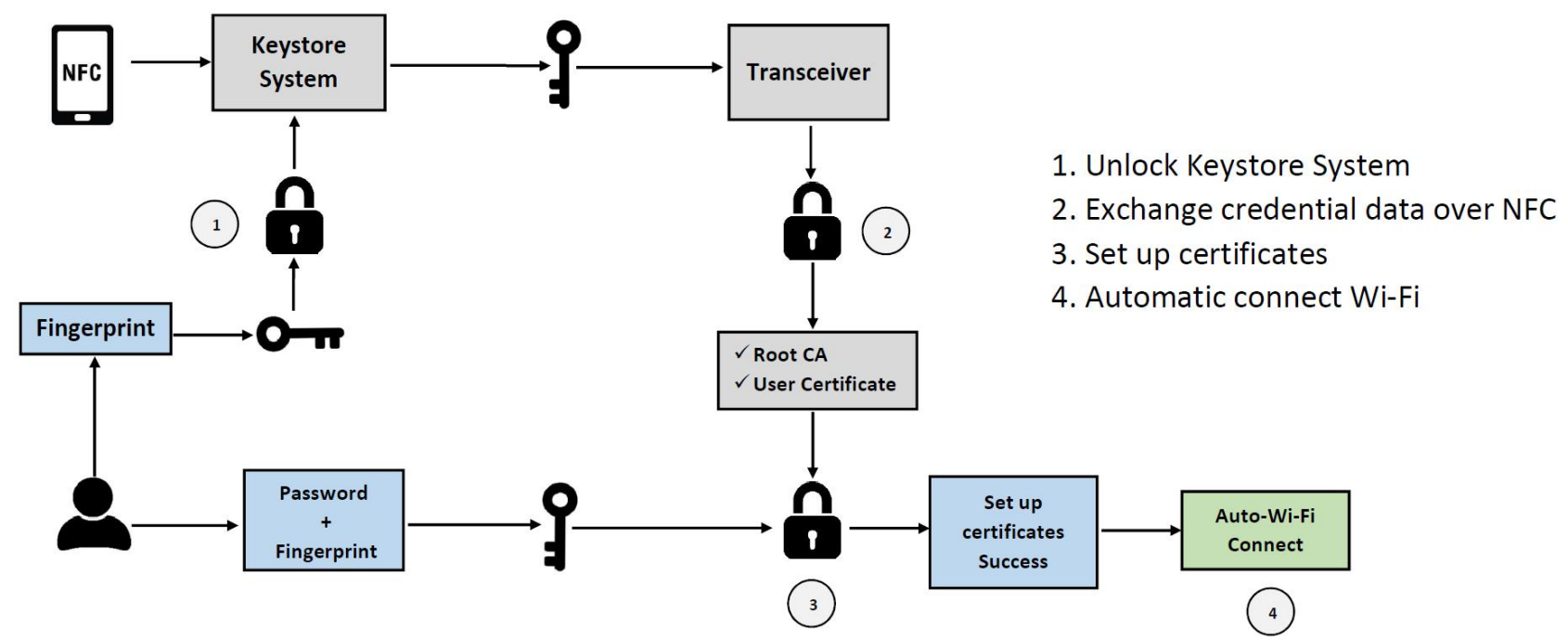

Fig. 2: Workflow of authentication process.

- Unlock user's Keystore System

A user taps fingerprint on device's sensor to unlock the Keystore system on the phone. This step is considered the first factor in the authentication process as "what you are".

- Exchange credential data over NFC

Next the user brings the smartphone to touch NFC terminal. The NFC Java program running on RADIUS server will inquire the application identification (AID) on the target device. The server application then verifies whether the user data exists in the database. If found, the root certificate authority, user's certificate, and network information will be encrypted and sent to the client side. At this step, the data contained in 
Keystore are used for credentials requests to server, that is considered as "what you have". Example of the initialization before starting data exchange is illustrated in Fig. 3.

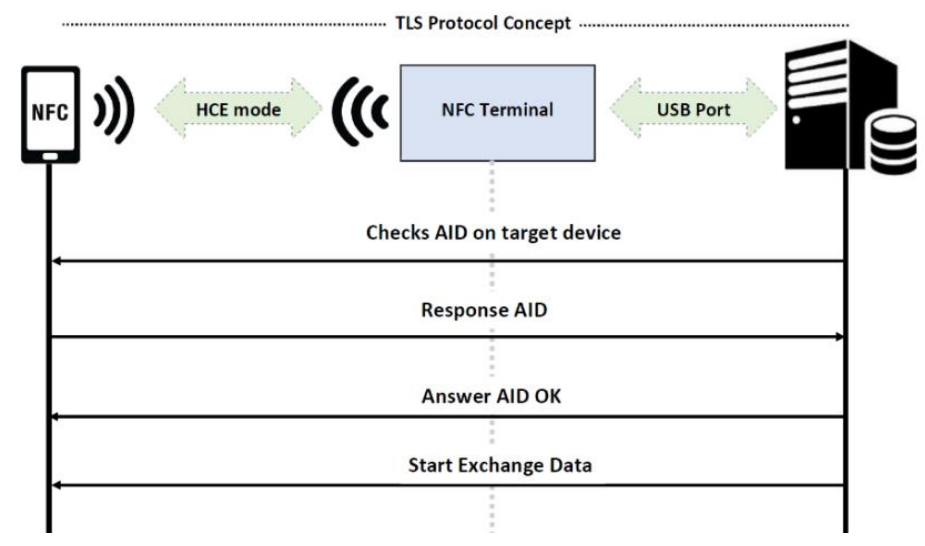

Fig. 3: Initialization before data exchange between Android and server over NFC.

\section{- Set up certificates}

Once the server has returned credentials data, the application on smartphone calls the certificate setup module to install certificates with pop-up notification, which is a vital step since the user must realize and confirm before importing certificates into their devices. At this step, it requires fingerprint/PIN to set up CA.crt as shown in Fig. 4 and password to extract the client certificate as shown in Fig. 5. This step represents as "what you know" and "what you are".

\section{- Automatic connect Wi-Fi}

Finally, the network manager function enforces the device to securely connect to the target wireless access point with prior network information obtained from the server sides, including service set identifier (SSID), security types, EAP method, and identity. The key advantage is to eliminate associate to rogue AP.
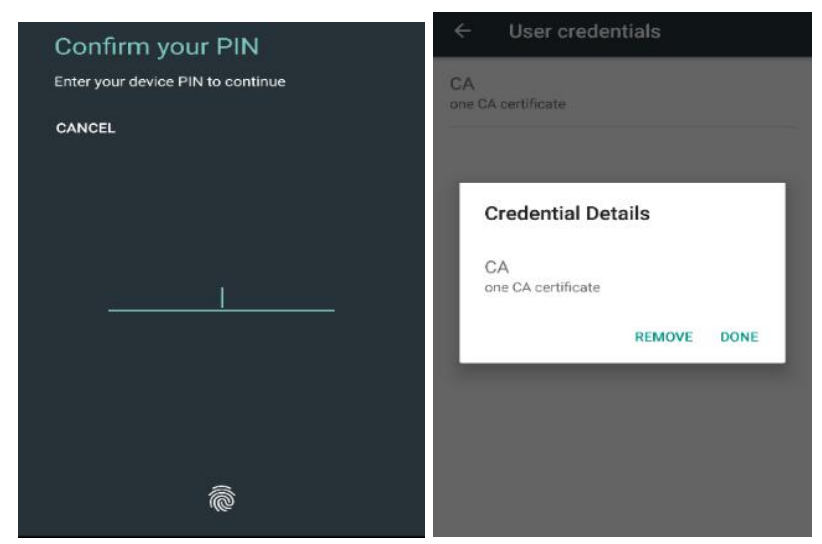

Fig. 4: Set up root CA certificate.
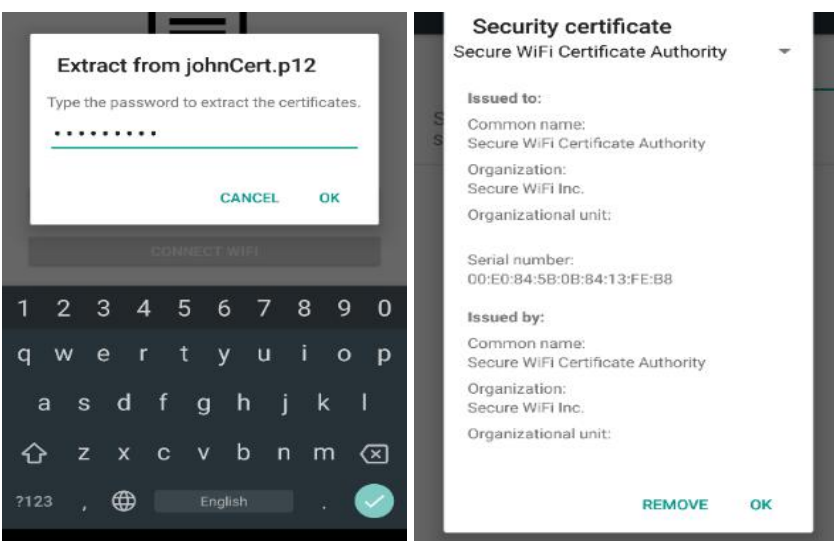

Fig. 5: Set up user certificate. 


\section{Experimental Result}

Table 1 illustrates the experiment result from 100 iterations at $95 \%$ of confidence interval. The outcome demonstrates that the exchange of credentials information somewhat consumes time more than connecting Wi-Fi and authentication component. However, this is comprehensible due to the fact that there is a latency of sub-processors like data encryption and decryption, certificate file sizes including uncertain performance's host-based card emulation. These factors significantly increase the task duration.

Table 1: Duration time of each component.

\begin{tabular}{l|lll}
\hline Components & Min $(s)$ & Max $(s)$ & Average $(s)$ \\
\hline Exchange credentials data over NFC & 3.657 & 5.028 & $3.856 \pm 0.0665$ \\
Connect to Wi-Fi \& Authentication & 3.235 & 3.352 & $3.254 \pm 0.0035$ \\
Total & 6.903 & 8.293 & $7.110 \pm 0.0673$ \\
\hline
\end{tabular}

\section{Conclusion}

The use of certificate-based authentication is a highly secure solution but one major drawback is the difficulty of certificate management. By leveraging the existing NFC chip on smartphones as certificate channel can ease the burden of the system administrator. Meanwhile, the multi-factor authentication ensures that each stage must be granted by the user from three independent categories. The findings from the preliminary experimental result on the prototype reported that the time spent on obtaining certificates and internet access can be achieved within a few seconds. Future direction would be the integration with roaming mechanism Hotspot $2.0(802.1 \mathrm{u})$ that could support for all EAP methods such as EAP-TTLS and MSCHAPv2.

\section{References}

[1] "Secure Wi-Fi is important. But secure IoT is vital." [Online]. Available: https://blog.avast.com/secure-wi-fiimportant-secure-iot-vital. [Accessed: 15-Oct-2017].

[2] “Bring your own device," Wikipedia. 09-Oct-2017.

[3] "What is BYOD and How Does It Impact Your Wireless Network?," 17-Feb-2016. [Online]. Available: https://www.taylored.com/blog/what-is-byod-and-how-does-it-impact-your-wireless-network/. [Accessed: 15-Oct2017].

[4] T. Radivilova and H. A. Hassan, "Test for penetration in Wi-Fi network: Attacks on WPA2-PSK and WPA2enterprise," in 2017 International Conference on Information and Telecommunication Technologies and Radio Electronics (UkrMiCo), 2017, pp. 1-4.

[5] V. Coskun, K. Ok, and B. Ozdenizci, Near Field Communication: From Theory to Practice. 2011.

[6] K. Abhishek, S. Roshan, P. Kumar, and R. Ranjan, "A Comprehensive Study on Multifactor Authentication Schemes," in Advances in Computing and Information Technology, 2013, pp. 561-568.

[7] "IEEE 802.1X-2010 - IEEE Standard for Local and metropolitan area networks--Port-Based Network Access Control." [Online]. Available: https://standards.ieee.org/findstds/standard/802.1X-2010.html. [Accessed: 30-Oct2017].

[8] M. Schukat and P. Cortijo, "Public key infrastructures and digital certificates for the Internet of things," in 2015 26th Irish Signals and Systems Conference (ISSC), 2015, pp. 1-5.

[9] A. Matos, D. Romão, and P. Trezentos, "Secure hotspot authentication through a Near Field Communication sidechannel," in 2012 IEEE 8th International Conference on Wireless and Mobile Computing, Networking and Communications (WiMob), 2012, pp. 807-814.

[10] C. Sudar, S. K. Arjun, and L. R. Deepthi, "Time-based one-time password for Wi-Fi authentication and security," in 2017 International Conference on Advances in Computing, Communications and Informatics (ICACCI), 2017, pp. 1212-1216. 